\title{
Etapas de cambio y regulación en usuarios de servicios deportivos. Relación con la satisfacción y la lealtad Stages of change and regulation in users of sports services. Relationship with satisfaction and loyalty
}

Luis Alberto Dueñas-Dorado*, José Alberto Pérez-García*, José Leandro Trístán-Rodríguez*, Flavia da CunhaBastos**, Alberto Nuviala***

* Universidad Autónoma de Nuevo León (México), **Universidad de Sao Paulo (Brasil), ***Universidad Pablo de Olavide Sevilla (España)

Resumen. La actividad física y su práctica regular contribuyen a mantener un estilo de vida activo y saludable. Los responsables de las organizaciones deportivas deben comprender el comportamiento de los usuarios actuales y potenciales para establecer estrategias de promoción y consolidación de la actividad física. El objetivo de este trabajo es averiguar si en usuarios de servicios deportivos se encuentran asociaciones entre etapas de cambio y tipos de regulación, así como diferentes niveles de satisfacción, lealtad y cantidad de práctica de actividad física realizada en relación a las etapas de cambio. La población objeto de estudio fueron 4236 usuarios inscritos en centros deportivos públicos municipales de Monterrey (México). La edad media es 19.30£12.65, siendo el 55.7\% hombres. Se utilizaron varios instrumentos para la investigación: el Behavioural Regulation in Exercise Questionnaire, Cuestionario de etapas de cambio, cuatro ítems del EPOD2, y cuatro ítems de una escala de intenciones futuras de usuarios servicios deportivos. Todos ellos contaban con la validez y fiabilidad necesarias. Se realizaron pruebas de comparación de medias, tablas de contingencia y correlaciones. Los resultados ponen de manifiesto que existe una asociación entre etapas de cambio, regulación, satisfacción, lealtad, tiempo medio de la sesión y frecuencia semanal de práctica, que los responsables de organizaciones, programas y actividades deportivas, deben conocer y utilizar para incrementar las tasas de práctica de actividad física y la consolidación del hábito entre los diferentes grupos poblacionales. Son los usuarios que se encuentran en las etapas de acción y mantenimiento los más satisfechos, los más leales y los que más actividad física realizan.

Palabrasclave: motivación, etapas de cambio, satisfacción, lealtad, cantidad de actividad física.

\begin{abstract}
Physical activity and regular practice help to maintain an active and healthy lifestyle. Sport organization leaders are to understand the behaviour of current and potential users to establish strategies for promoting and consolidating physical activity. The objective of this project is to discover whether sports service users find associations between stages of change and types of regulation, as well as different levels of satisfaction, loyalty and amount of physical activity practices completed out in relation to the stages of change. The population under study was 4236 users registered in municipal public sports centers in Monterrey (Mexico). The average age is $19.30 \pm 12.65$, with $55.7 \%$ males. Several instruments were used for the research: the Behavioural Regulation in Exercise Questionnaire, Stages of Change Questionnaire, four items from EPOD2, and four items from a scale of future intentions of users of sports services. All of them had the necessary validity and reliability. Mean comparison tests, contingency tables and correlations were performed. The results show that there is a partnership between stages of change, regulation, satisfaction, loyalty, average session time and weekly frequency of practice, which those responsible for organizations, programs and sports activities, should be aware of and use to increase rates of physical activity practice and the consolidation of habit among different population groups. It is the users who are in the stages of action and maintenance the most satisfied, the most loyal and the ones that perform the most physical activity.
\end{abstract}

Keywords: motivation, stages of change, satisfaction, loyalty, quantity of physical activity performed.

\section{Introducción}

La actividad física y su práctica regular son conductas que resguardan contra muchas enfermedades, previenen el aumento de peso y contribuyen a mantener un estilo de vida saludable (Mujika-Alberdi, GibajaMartins, \& García-Arrizabalaga, 2021). Por el contrario, la inactividad física es uno de los principales facto-

Fecha recepción: 14-09-20. Fecha de aceptación: 24-11-20

Luis Alberto Dueñas Dorado

luis.duenasdrd@uanl.edu.mx res de riesgo para todo tipo de enfermedades, especialmente el sobrepeso, relacionado con múltiples enfermedades, según World Health Organization (WHO) (2004). En México, por ejemplo, siete de cada 10 adultos padecen sobrepeso u obesidad según datos del Instituto Nacional de Salud Pública (INSP, 2016). Por todo ello es indispensable que los responsables de las organizaciones, tanto públicas como privadas relacionadas con la actividad física y el deporte, comprendan las razones y/o circunstancias que rodean el inicio, mantenimiento y continuidad de este hábito y conozcan cómo las personas pueden cambiar de forma intencionada sus hábitos 
de vida y práctica de actividad física.

Para comprender los comportamientos de la población en relación a la actividad física y el deporte, es necesario entender la incidencia que la motivación tiene sobre ella (Nuviala, Gómez-López, Grao-Cruces, Granero-Gallegos, \& Nuviala, 2013). La motivación es entendida como el producto de una serie de variables sociales, ambientales e individuales que determinan la elección de una actividad física o deportiva, la intensidad en la práctica de esa actividad, la persistencia en la tarea, el rendimiento, los resultados deportivos obtenidos, los aspectos que influyen a que un individuo se inscriba en una instalación u otra, o determina que permanezca en un centro deportivo o lo abandone (Boceta, 2012; Moreno, Cervelló, \& Martínez, 2007). La influencia de los procesos motivacionales sobre la práctica y adherencia de actividades físico-deportivas constituyen un tema ampliamente estudiado (Jiménez, Moreno, Leyton, \& Claver, 2015; Murillo et al., 2018;Zamarripa, Castillo, Baños, Delgado, \& Álvarez, 2018) que se han desarrollado desde dos perspectivas teóricas por separado: el modelo transteórico de cambio conductual (Prochaska \& DiClemente, 1982) y la teoría de la autodeterminación (Deci \& Ryan, 1985; Ryan \& Deci, 2000).

El modelo transteórico (MTT) está conformado de dos conceptos principales: las etapas y los procesos de cambios. Las etapas explican el cuándo y los procesos el cómo se producen los cambios en las actitudes, intenciones y comportamientos de los individuos (Marcus \& Forsyth, 2009). Este modelo apoya la idea de que el ejercicio físico es un comportamiento dinámico, y que las personas pasan por cinco etapas de comportamiento en sus intentos de cambiar su estilo de vida sedentario en uno que sea físicamente más activo (Zamarripa et al., 2018). Las etapas incluidas en el modelo son precontemplación (el sujeto no realiza actividad física y no tiene intención de realizarlo), contemplación (el sujeto está inactivo y tiene intención de cambiar), preparación (el sujeto está activo pero no lleva a cabo las recomendaciones para una práctica saludable), acción (el sujeto está activo y cumple con las recomendaciones para una práctica saludable, pero no ha completado seis meses completos de práctica regular) y por último el mantenimiento (la persona ha realizado una actividad física saludable durante más de 6 meses). Son las etapas del cambio del ejercicio de acción y mantenimiento las más activas (Aspano, Lobato, Leyton, Batista, \& Jiménez, 2016; Zamarripa et al., 2018).

Por su parte, los procesos de cambio, son actividades visibles y no visibles que modifican experiencias y el ambiente de los individuos, a los que se recurre para cambiar la conducta. Se clasifican en dos grupos: procesos de cambio de la experiencia (toma de conciencia; liberación dramática; reevaluación ambiental; autoevaluación; liberación social) y procesos de cambio del ambiente (contra condicionamiento; relaciones de ayuda; manejo de los reforzadores; autoliberación; control de estímulos) (Nigg \& Courneya, 1998). Rodgers, Courneya y Bayduza (2001) concluyen en su trabajo que los procesos del ambiente se incrementan hasta la etapa de mantenimiento.

La transición entre las distintas etapas de cambio en el individuo radica en la motivación (Jiménez et al., 2015), pasando las personas por las etapas no necesariamente de manera lineal (Gallegos-Sánchez, Ruiz-Juan, Villarreal-Angeles, \& Zamarripa-Rivera, 2019). Estas etapas de cambio guardan relación con la teoría de la autodeterminación (TAD). Esta ha sido la teoría motivacional que más relevancia ha cobrado en los últimos años en el ámbito del ejercicio físico y se emplea para explicar el fenómeno de la adherencia a la práctica deportiva (González-Cutre, Sicilia, \& Fernández, 2010; Muyor, Águila, Sicilia, \& Orta, 2009). La TAD considera que la motivación es un continuo que abarca diferentes grados de autodeterminación de la conducta (Muyor, et al., 2009), con comportamientos que van desde altos niveles de autonomía (motivación intrínseca) hasta niveles medios (motivación extrínseca) y niveles bajos (no motivación o amotivación) (Deci \& Ryan, 1985).

Existen estudios que han establecido una relación entre las etapas de cambio y la motivación. Estudios como los de Rose, Parfitt yWilliams (2005), ThogersenNtoumani y Ntoumanis (2006) o Buckworth, Lee, Regan, Schneider y DiClemente (2007), concluyeron que las personas que se encuentran en las etapas del cambio de acción y mantenimiento, parecen tener una motivación más autodeterminada que el resto de etapas. Con población mexicana, Zamarripa et al. (2018) concluyeron que las regulaciones menos autodeterminadas (externas y desmotivación) predominaron en las primeras etapas de cambio (pre-contemplación y contemplación) y disminuyeron en las últimas etapas (acción y mantenimiento); en contraste, las regulaciones más autodeterminadas (intrínsecas, integradas e identificadas) predominaron en las últimas etapas (acción y mantenimiento) y fueron menores en las primeras etapas (precontemplación y contemplación). Sin embargo, en la literatura no aparecen estudios que relacionen motivación y etapas de cambio en usuarios y clientes de servicios deportivos. 
La adherencia a la actividad física, además de tener relación con la motivación y las etapas de cambio, también se relaciona con otros constructos como son la satisfacción y la lealtad con el servicio deportivo recibido. Diferentes estudios (Bernal, 2014; Boceta, 2012; PérezAmate, 2015), tesis doctorales, ponen de manifiesto que una mayor satisfacción con el servicio deportivo o actividad física realizada, se corresponde con una mayor frecuencia y cantidad de práctica deportiva, no habiéndose publicado artículos científicos con estos objetivos. La satisfacción, por su parte, es un constructo esencial para desarrollar estrategias de fidelización y retención de clientes (Cronin, Brady, \& Hult, 2000). Oliver (1997) definió la satisfacción como el cumplimiento placentero de una necesidad, deseo o meta después de consumir un producto o servicio. La satisfacción es el resultado de percepciones tanto cognitivas como afectivas (Li \& Petrick, 2010). La lealtad se entiende como una actitud favorable del cliente hacia el servicio deportivo, en términos de recomendar el centro y sus servicios, y mostrar comportamientos de recompra positivos (Yoshida \& James, 2010). La investigación sobre servicios deportivos y de fitness ha puesto de manifiesto la relación satisfacción y lealtad (Fernández-Martínez, HaroGonzález, Nuviala, Pérez-Ordás, \& Nuviala, 2020; Pérez-Ordás, Aznar-Cebamanos, Nuviala, \& Nuviala, 2019). Si un cliente está satisfecho, hay más posibilidades de que el cliente tenga una percepción positiva de la organización y demuestre lealtad a esa entidad (GarcíaFernández et al., 2018).

Sin embargo, a pesar de estas evidencias, en la literatura no se encuentran estudios que relacionen etapas de cambio, motivación, satisfacción y lealtad de usuarios o clientes de servicios deportivos, a pesar de la importancia que este conocimiento tiene para los responsables de la dirección de organizaciones deportivas y para establecer estrategias de consolidación de práctica de actividad física y deportiva. Por ello, este trabajo pretende averiguar si en usuarios de servicios deportivos se encuentran asociaciones entre etapas de cambio y niveles de regulación motivacional, así como diferentes niveles de satisfacción, lealtad y cantidad de práctica de actividad física realizada en relación a las etapas de cambio.

\section{Material y métodos}

\section{Participantes}

La población objeto de estudio estaba conformada por 4236 usuarios inscritos en 27 centros deportivos públicos municipales de la zona metropolitana de Monterrey, Nuevo León (México). Se realizó una selección aleatoria por centros. El conjunto de características que conforman el perfil de los sujetos estudiados muestra una población en su mayoría hombres (55.7\%). La edad oscila entre los 12 a los 84 años ( $M$ edad $=$ $19.30 \pm 12.65)$. El porcentaje mayor lo conformaban estudiantes con 3149 usuarios (74.3\%), seguidos de trabajadores $543(13.5 \%)$ y por último $544(12.1 \%)$ que desempeñaban otras actividades (Jubilados/pensionados, desempleados, trabajos domésticos y otros).

\section{Instrumentos}

Para la recolección de la información, se utilizó el BREQ-3 (Behavioural Regulation in Exercise Questionnaire), desarrollado por Markland y Tobin (2004), modificada por Wilson, Rodgers, Loitz y Scime (2006). Posteriormente fue traducido y adaptado al español por González-Cutre et al. (2010), cuyo objetivo es el de comprobar los motivos de práctica deportiva de usuarios de organizaciones y actividades deportivas de tiempo libre. El instrumento está formado con un total de 23 ítems y seis tipos de regulación: motivación intrínseca (porque creo que el ejercicio es divertido....); motivación integrada (porque está de acuerdo con mi forma de vida....); motivación identificada (porque valoro los beneficios que tiene el ejercicio físico...); motivación introyectada (porque me siento culpable cuando no lo practico...); regulación externa (porque los demás me dicen que debo hacerlo...); desmotivación(no veo por qué tengo que hacerlo...).

Para indagar en qué etapa de cambio se encontraba el usuario de servicios deportivos públicos municipales, se aplicó el Cuestionario de etapas de cambio de Marcus y Forsyth (2009) adaptado y validado al contexto mexicano por Zamarripa, Ruiz-Juan y Ruiz-Risueño (2019), el cual consiste en una breve definición del concepto de actividad física y actividad física regular, además de cuatro preguntas las cuales se responden de forma dicotómica (sí o no) en las siguientes afirmaciones; (1) actualmente estoy físicamente activo; (2) tengo la intención de ser más activo físicamente en los próximos 6 meses, (3) actualmente, hago clases regulares, actividad física; y (4) he estado haciendo actividad física regular durante los últimos 6 meses. Estudios previos han confirmado la fiabilidad y validez de criterio (Marcus \& Forsyth, 2009; Zamarripa et al., 2019).

Para conocer la satisfacción de los usuarios se utilizaron cuatro ítems de EPOD2 (Nuviala et al., 2013) que van de 1 a 5. Los ítems utilizados son: «Haber elegido 
esta organización ha sido una buena decisión»; «Estoy conforme por haberme matriculado/inscrito en esta organización»; «Tuve una buena idea al decidir incorporarme a realizar actividades deportivas en esta organización»; «Estoy complacido por haberme matriculado en esta actividad».

La lealtad fue medida a través de cuatro ítems utilizados en una escala de intenciones futuras de usuarios servicios deportivos que oscila entre 1 y 7 (Nuviala et al., 2014). Los ítems utilizados fueron: «Contaré aspectos positivos sobre el club deportivo a otras personas»; «Recomendaré el centro deportivo a cualquiera que busque mi consejo»; «Animaré a mis amigos y familiares a hacer actividades deportivas en este centro»; «Para cualquier servicio deportivo que pudiera necesitar, consideraré este club como la primera opción»; «En los próximos años realizaré más actividades deportivas en este club».

\section{Procedimiento}

El trabajo, descriptivo, observacional y transversal, fue realizado en México durante el primer trimestre del año 2018, dirigido por el equipo que firma este trabajo, todos ellos Licenciados en Ciencias de la Actividad Física y el Deporte. Antes de proceder a la recogida de datos, se envió una carta de solicitud de permiso a los responsables de los diferentes centros deportivos municipales que participaron en el estudio (San Nicolás de los Garza, Monterrey, Escobedo y Guadalupe). Posteriormente se solicitó a los participantes que lo respondieran de forma voluntaria y que consultaran cualquier duda que tuvieran con los ítems. En el caso de los menores se informó a los padres y se solicitó su consentimiento. Todos los participantes fueron informados de las medidas de seguridad pertinentes para proteger el anonimato, imagen y confidencialidad de todos los datos, y que no existían respuestas correctas o incorrectas, verdaderas o falsas y aceptaron contestar al cuestionario. También se les pidió que respondieran con sinceridad y honestidad.

Los usuarios que colaboraron tardaron aproximadamente 10 minutos en facilitar los datos que se solicitaban en los cuestionarios. En la recepción de los centros deportivos municipales, donde se facilitaban las encuestas, había un responsable para supervisar y resolver las posibles cuestiones que pudieran surgir.

La presente investigación se realizó de acuerdo con las pautas éticas internacionales, que son consistentes con las directrices de la
Asociación Americana de Psicología (APA). También se tuvieron en cuenta en todo momento los principios fundamentales establecidos en la Declaración de Helsinki (revisada en 2013, Brasil). Se obtuvo la aprobación ética para el estudio de la ética de la Universidad Autónoma de Nuevo León (México) comité de revisión (No. 16CI19039021). De la misma forma el trabajo cuenta con el visto bueno del Vicerrectorado de Investigación y Transferencias de la Universidad Pablo de Olavide de Sevilla.

\section{Análisis estadístico}

En primer lugar, se constató la consistencia interna de los instrumentos utilizados mediante el cálculo de la confiabilidad compuesta (FC) y la varianza promedio extraída (AVE). También se calculó la correlación entre los diferentes constructos que conforman el instrumento. Se calcularon los porcentajes de las etapas de cambio de los usuarios de servicios deportivos. Se realizó la prueba de ANOVA para comprobar la existencia de diferencias en relación a la satisfacción, intenciones futuras y tiempo de práctica medio por sesión en función de las etapas de cambio. Se realizó una prueba de contingencia para estudiar la asociación entre etapa de cambio y frecuencia de práctica semanal. De igual forma se estudiaron las variaciones en las diferentes regulaciones a través de las etapas de mediante análisis de varianza multivariante (MANOVA). Los cálculos se realizaron con el programa SPSS 22.

\section{Resultados}

Se procedió en primer lugar a constatar la confiabilidad de los instrumentos. La fiabilidad compuesta (FC) varió entre .81 y 82. Los valores de la varianza promedio extraída (AVE) están entre .52 y .76. Los resultados demuestran la consistencia de los instrumentos utilizados en la investigación (Tabla 1).

Las correlaciones entre los diferentes constructos que integran la investigación muestran una clara relación entre satisfacción y lealtad. De la misma forma se
Tabla 1

Media, desviación estándar, Alfa de Cronbach, varianza media extraída, fiabilidad compuesta y correlaciones

\begin{tabular}{|c|c|c|c|c|c|c|c|c|c|c|c|c|}
\hline & Media & S.D. & $\alpha$ & AVE FC & 1 & 2 & 3 & 4 & 5 & 6 & 7 & 8 \\
\hline 1. Satisfacción & 3.94 & .90 & .896 & .76 .92 & - & $.580^{* *}$ & $.272^{* *}$ & $.264^{* *}$ & $.239^{* *}$ & $.052^{* *}$ & $-.035^{*}$ & -.010 \\
\hline 2. Lealtad & 5.54 & 1.16 & .891 & .69 .91 & & & $.328^{* *}$ & $.326^{* *}$ & $.299^{* *}$ & $.081^{* *}$ & $-.032^{*}$ & -.007 \\
\hline 3. Regul. intrínseca & 4.07 & .75 & .726 & .54 .83 & & & & $.715^{* *}$ & $.658^{* *}$ & $.011^{* *}$ & $-.152^{* *}$ & $-.210^{* *}$ \\
\hline 4. Regul. integrada & 3.92 & .80 & .748 & .56 .82 & & & & & $.672^{* *}$ & $.103^{* *}$ & $-.070^{* *}$ & $-.116^{* *}$ \\
\hline 5. Regul. identificada & 4.06 & .77 & .703 & $.61 \quad .82$ & & & & & & $.062^{* *}$ & $-.139^{* *}$ & $-.156^{* *}$ \\
\hline 6. Regul. introyectada & 2.72 & .96 & .721 & $.54 \quad .82$ & & & & & & & $.585^{* *}$ & $.456^{* *}$ \\
\hline 7. Regul. externa & 2.32 & 1.02 & .766 & .58 .85 & & & & & & & & $.614^{* *}$ \\
\hline 8. Amotivación & 2.36 & .97 & .712 & .52 .81 & & & & & & & & \\
\hline Tiempo medio sesión & 103.85 & 65.66 & - & $-\quad-$ & $.042^{* *}$ & $.074^{* *}$ & $.093^{* *}$ & $.120^{* *}$ & $.125^{* *}$ & $.034^{*}$ & -.026 & -.007 \\
\hline
\end{tabular}


observa correlación entre estos dos constructos y las dimensiones incluidas en BREQ-3, excepto amotivación. Las dimensiones incluidas en BREQ-3 correlacionan todas entre sí, con valores pequeños, excepto las correlaciones entre motivación intrínseca, motivación integrada y motivación identificada y las correlaciones entre amotivación, motivación externa y motivación introyectada. Destacar que regulación externa y amotivación se correlacionaron positivamente entre sí y negativamente con las motivaciones más autónomas. Estos resultados proporcionan evidencia de validez discriminante entre las subescalas que conforman el estudio.

Casi un 85\% de los usuarios de servicios deportivos públicos se encuentran es las etapas más estables de consolidación de su práctica deportiva. La satisfacción media es de casi 4 puntos sobre 5 y la lealtad a los servicios deportivos es de 5.54 sobre 7 (Tabla 1). Existen diferencias significativas entre la etapa de cambio y la satisfacción con el servicio recibido. Son los usuarios que se encuentran en las etapas de acción y mantenimiento los más satisfechos con el servicio recibido. De la misma forma los más leales a la organización deportiva son los usuarios que se encuentran en las etapas de acción y mantenimiento (Tabla 2).

Tabla 2

Etapas de cambio, regulación, satisfacción, lealtad, tiempo medio por sesión y frecuencia de práctica semanal. Media, desviación típica, Manova, tamaño del efecto, Anova y tabla de contingencia

\begin{tabular}{lccccccc}
\hline \multicolumn{7}{c}{ Etapa de cambio } \\
\hline Variables & $\begin{array}{c}\text { Precontemplación } \\
(2.2 \%)\end{array}$ & $\begin{array}{c}\text { Contemplación } \\
(8.3 \%)\end{array}$ & $\begin{array}{c}\text { Preparación } \\
(6.1 \%)\end{array}$ & $\begin{array}{c}\text { Acción } \\
(14.0 \%)\end{array}$ & $\begin{array}{c}\text { Mantenimiento } \\
(69.4 \%)\end{array}$ & $\mathrm{F}$ & $\mu 2$ \\
\hline Regulac. Intrínseca & $3.63 \pm .80$ & $3.89 \pm .77$ & $3.93 \pm .74$ & $3.98 \pm .72$ & $4.13 \pm .74$ & $23.151^{* *} .021$ \\
Regulac. Integrada & $3.43 \pm .91$ & $3.61 \pm .78$ & $3.70 \pm .77$ & $3.76 \pm .79$ & $4.02 \pm .78$ & $45.240^{* *} .041$ \\
Regulac. Identificada & $3.63 \pm .85$ & $3.93 \pm .74$ & $3.89 \pm .74$ & $3.98 \pm .71$ & $4.12 \pm .78$ & $19.378^{* *} .018$ \\
Regulac. Introyectada & $2.68 \pm .93$ & $2.76 \pm .99$ & $2.90 \pm .96$ & $2.71 \pm .96$ & $2.70 \pm .96$ & $2.673^{*} .003$ \\
Regulac. Externa & $2.56 \pm .91$ & $2.54 \pm 1.08$ & $2.60 \pm 1.10$ & $2.28 \pm .94$ & $2.27 \pm 1.02$ & $12.013^{* *} .011$ \\
Amotivación & $2.61 \pm .92$ & $2.41 \pm 1.05$ & $2.58 \pm 1.04$ & $2.33 \pm .90$ & $2.33 \pm .97$ & $5.891^{* *} .006$ \\
\hline Satisfacción & $3.70 \pm 1.07$ & $3.80 \pm .89$ & $3.67 \pm .97$ & $3.88 \pm .86$ & $4.00 \pm .89$ & $13.099^{* *} .020$ \\
Lealtad & $5.06 \pm 1.39$ & $5.30 \pm 1.16$ & $5.18 \pm 1.28$ & $5.40 \pm .1 .13$ & $5.65 \pm 1.38$ & $22.678^{* *} .031$ \\
\hline Tiempo sesión & $94.30 \pm 8.58$ & $89.53 \pm 8.02$ & $95.18 \pm 7.43$ & $95.71 \pm 9.37$ & $108.25 \pm 8.70$ & $11.450^{* *} .047$ \\
\hline & Precontemplación & Contemplación & Preparación & Acción & Mantenimiento & $X^{2}$ \\
\hline 1 vez por semana & $14.0 \%$ & $8.3 \%$ & $5.8 \%$ & $1.9 \%$ & $1.4 \%$ & \\
2 veces por semana & $24.7 \%$ & $20.6 \%$ & $30.8 \%$ & $9.3 \%$ & $11.7 \%$ & $252.975^{* *}$ \\
3 veces por semana & $32.3 \%$ & $37.1 \%$ & $33.5 \%$ & $46.8 \%$ & $41.1 \%$ & 25 \\
4 o más veces por semana & $29.0 \%$ & $34.0 \%$ & $30.0 \%$ & $42.1 \%$ & $45.8 \%$ & \\
\hline
\end{tabular}

Cuando se analiza las diferentes regulaciones en cada una de las etapas, puede observarse en los resultados la existencia de diferencias significativas en todas ellas. Por regla general los resultados muestran que las regulaciones más autónomas presentan valores más altos en las etapas de cambio más consolidadas en relación al ejercicio. Por el contrario, en las etapas menos consolidadas de ejercicio físico, aparecen valores más altos de amotivación y regulación externa (Tabla 2; Figura 1).

La Tabla 2 muestra como el tiempo medio por sesión es diferente, siendo superior al resto en la etapa de mantenimiento. Son los usuarios que se encuentran en

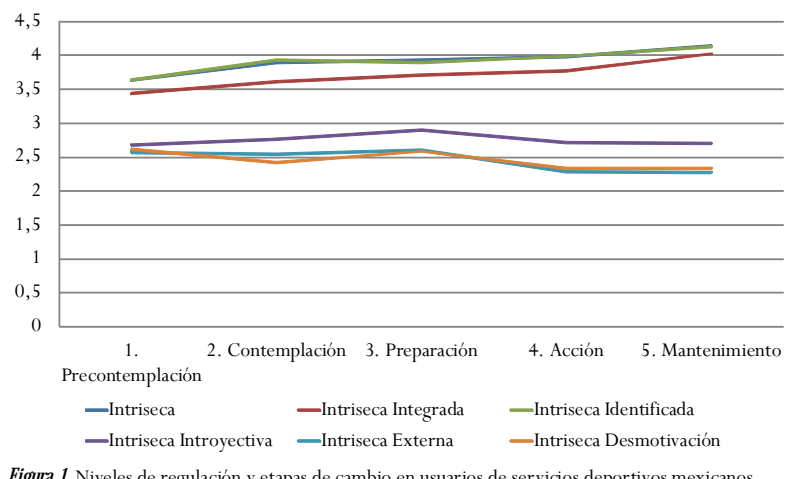

las etapas de precontemplación y contemplación, quienes realizan sesiones más cortas. De igual forma, los porcentajes de usuarios que asiste más veces por semana a los centros deportivos se encuentra entre los usuarios incluidos en las etapas de acción y mantenimiento.

\section{Discusión}

El fin de este artículo es estudiar si existía relación entre las etapas de cambio, motivación, satisfacción y lealtad de usuarios o clientes de servicios deportivos, puesto que en la literatura no se han encontrado trabajos sobre esta temática, a pesar de la importancia que este conocimiento tiene para los responsables de la dirección de organizaciones deportivas con el objeto de establecer estrategias de consolidación de práctica de actividad física y deportiva. Los objetivos planteados eran averiguar si en usuarios de servicios deportivos se encuentran asociaciones entre etapas de cambio y niveles de regulación motivacional, así como diferentes niveles de satisfacción, lealtad y cantidad de práctica de actividad física realizada en relación a las etapas de cambio.

En un primer momento se constató la bondad de los instrumentos utilizados para tal fin. Se constató la fiabilidad y validez de cada uno de ellos. Los resultados muestran correlaciones significativas entre los constructos que conforman la investigación. Los cálculos de FC y AVE, presentaron valores adecuados, que para el caso de AVE, estuvieron comprendidos entre .52 y .76 , resultados superiores a .5 (Hair, Black, Babin, Anderson, \& Tatham, 2006). Por lo que respecta a FC estuvieron entre .81 y .92, lo que suponen valores superiores a .6 (Bagozzi \& Yi, 1988), mínimo para ser consideradores adecuados. Resultados que ponen en valor la validez y fiabilidad del instrumento utilizado. 
El primero de los objetivos del presente estudio fue analizar la asociación de las etapas de cambio y las diferentes regulaciones. Los resultados han puesto en valor que la motivación está relacionada con las etapas de cambio en usuarios de servicios deportivos mexicanos. Es decir, a medida que la motivación de los usuarios se vuelve más autodeterminada, su disposición a realizar ejercicio físico se vuelve más constante. Así, los usuarios ubicados en las primeras etapas de cambio presentan valores más altos de desmotivación y la regulación externa. Por el contrario, los usuarios ubicados en las etapas de acción y mantenimiento, presentan valores más altos en las regulaciones intrínsecas, integradas e identificadas. Estos resultados coinciden con diversos estudios, siempre realizados en poblaciones que no se caracterizaban por ser usuarios identificados de centros deportivos y/o de actividad física dirigida. Así, Rose et al. (2005), Thogersen-Ntoumani y Ntoumanis (2006), Buckworth et al. (2007) y Zamarripa et al. (2018), concluyeron que las personas que se encuentran en las etapas del cambio de acción y mantenimiento, parecen tener una motivación más autodeterminada que el resto de etapas. Todo ello viene a confirmar la asociación etapas de cambio y motivación.

Otro de los objetivos de este trabajo era comprobar si la cantidad de actividad física diaria y la frecuencia semanal, tenían diferencia en función de las etapas de cambio. Los resultados han demostrado que los usuarios que se encuentran en las etapas de cambio de acción y mantenimiento tienen una frecuencia semanal y un tiempo medio por sesión, superiores al resto de usuarios incluidos en otras etapas. Este resultado es lógico puesto que según la consideración cuantitativa que Daley y Duda (2006) hacen del modelo transteórico, debe existir una relación entre la cantidad de actividad física realizada y las etapas superiores. Es necesario recordar que, para estar incluido en la etapa de mantenimiento, los usuarios deben ser activos y haberlo sido durante los últimos seis meses, lo que implica un nivel considerable de actividad física. Es preciso no olvidar que los usuarios incluidos en las etapas más consolidadas de actividad física, presentan valores más elevados de motivación autodeterminada. En la Tabla 1, se ha visto que existe correlación entre motivación autodeterminada y cantidad de actividad física diaria realizada. Resultados que, apoyados en el trabajo de Leyton, Batista y JiménezCastuera (2020) realizado con 214 sujetos de ambos sexos con una edad de 16.46士.96, según el cual la motivación intrínseca está relacionada directamente con la intención de ser activo, vienen a corroborar la relación entre las variables analizadas en este trabajo. Estos resultados ponen de manifiesto que existe relación entre motivación, etapas de cambio, cantidad de actividad física diaria, frecuencia de práctica semanal y la intención de seguir siendo activo.

La satisfacción y lealtad con el servicio o actividad deportiva recibidas son otra de las estrategias para consolidar la práctica de actividad física en la población. Algunos trabajos (García-Pascual, Alguacil, \& MolinaGarcía, 2020) han puesto de manifiesto la relación satisfacción y lealtad, otros, además relacionaron ambas variables con la duración media de las sesiones y/o la frecuencia semanal de práctica organizada (Bernal, 2014; Boceta, 2012; Pérez-Amate, 2015). En este trabajo puede verse la correlación entre el tiempo medio por sesión y los constructos satisfacción y lealtad. Además, también puede observarse que los grupos que más frecuencia de práctica semanal son los grupos con satisfacción y lealtad mayores. Estudios como el de Nuviala, Pérez-Turpin, Tamayo-Fajardo y Fernández-Martínez (2011), realizado con población en edad escolar, ya habían manifestado que una mayor satisfacción con el servicio deportivo, suponía mayores tasas de práctica en la población. A todo ello hay que unir las conclusiones de Leyton, Batista, Lobato y Jiménez (2019) en un trabajo realizado con 812 personas de edades comprendidas entre los 14 y los 60 años (29.5 \pm 21.7$)$, quienes han puesto de manifiesto la relación entre las etapas de acción y mantenimiento, con la intención de seguir siendo activos, por lo que parece existir una relación entre etapas de cambio, regulación e intención de seguir siendo activo. Por ello, un incremento de la satisfacción y lealtad supone una estrategia para la promoción de la actividad física y el deporte.

Las etapas de cambio y la regulación, muestran de la misma forma una asociación con la frecuencia de práctica y el tiempo medio por sesión. Son los usuarios ubicados en las etapas de acción y mantenimiento los que presentan una mayor frecuencia de práctica semanal y un mayor tiempo medio por sesión. Este resultado viene a poner en valor la necesidad de incrementar los tiempos y la frecuencia de práctica como estrategias de consolidación de la práctica de actividad física, a través de la adecuación de los programas y actividades físicas de las organizaciones deportivas a las necesidades y expectativas de los usuarios (Nuviala et al., 2013).

\section{Conclusiones}

El trabajo aquí presentado permite afirmar que exis- 
te una asociación entre etapas de cambio, regulación, satisfacción, lealtad, tiempo medio de la sesión y frecuencia semanal de práctica, que los responsables de organizaciones, programas y actividades deportivas, deben conocer y utilizar para incrementar las tasas de práctica de actividad física y la consolidación del hábito entre los diferentes grupos poblacionales. Es necesario seguir investigando al respecto y diferenciar por variables sociodemográficas y sociodeportivas con fin de aproximar la oferta a los intereses y necesidades de la población.

\section{Referencias}

Aspano, M., Lobato, S., Leyton, M., Batista, M., \& Jiménez, R. (2016). Predicción de la motivación en los estadios de cambio de ejercicio más activos. Retos, 30 (30), 87-91. doi: 10.47197/retos.v0i30.40619

Bagozzi, R. P., \&Yi,Y. (1988). On the evaluation of structural equation models. J ournal of theAcademy of M arketing Science, 16(1), 74-94. doi :10.1007/BF02723327

Bernal, A. (2014). Fidelización de clientes en organizaciones de portivas: Calidad, valor percibido y satisfacción como factores determinantes. (Tesis doctoral). Universidad de Sevilla, Sevilla. http: / /hdl.handle.net/11441/53492

Boceta, M. (2012). Calidad percibida, satisfacción y valor percibido por los usuarios de servicios prestados por el instituto municipal de deportes del ayuntamiento de Sevilla en centros deportivos de gestión directa. Segmentación de usuarios (Tesis doctoral). Universidad Pablo de Olavide, Sevilla.

Buckworth, J., Lee, R. E., Regan, G., Schneider, L. K., \& DiClemente, C. C. (2007). Decomposing intrinsic and extrinsic motivation for exercise: Application to stages of motivational readiness. Psychology of Sport and Exercise, 8 (4), 441-461. doi.org/10.1016/j.psychsport.2006.06.007

Cronin, J., Brady, M.K., \& Hult, G. (2000). Assessing the effects of quality, value, and customer satisfaction on consumer behavioral intentions in service environments. Journal of Retailing, 76(2), 193-218. doi.org/10.1016/ S0022-4359(00)00028-2

Daley,A. J., \& Duda, J. L. (2006). Self-determination, stage of readiness to change for exercise, and frequency of physical activity in young people. European Journal of Sport Science, 6(4), 231-243. doi:10.1080/17461390601012637

Deci, E. L., \& Ryan, R. M. (1985). The general causality orientations scale: Self-determination in personality. Journal of research in personality, 19(2), 109-134. doi.org/10.1016/ 0092-6566(85)90023-6

Declaración de Helsinki de la asociación Médica Mundial. Principios éticos para las investigaciones médicas en seres humanos, 2013 [citado 22/09/2018]. Disponible en: h t t p : / / j a m a . ja m a n e twork. com / article.aspx?articleID $=1760320$
Fernández-Martínez, A., Haro-González, M., Nuviala, R., Pérez-Ordás, R. \& Nuviala, A. (2020). Women and Physical Activity in Fitness Centres. Analysis of Future Intentions and Their Relationship with Age. International Journal of Envi-ronmental Research and Public H ealth 17(15), 5289. https://doi.org/10.3390/ijerph17155289

Gallegos-Sánchez, J., Ruiz-Juan, F., Villarreal-Angeles, M., \& Zamarripa-Rivera, J. I. (2019). Etapas de cambio en la práctica de actividad física de tiempo libre en estudiantes de secundaria de Victoria de Durango, México. Retos, 35 (35), 196-200. https://doi.org/10.47197/ retos.v0i35.66878

García-Fernández, J., Gálvez-Ruíz. P., Fernández-Gavira, J., Vélez-Colón, L., Pitts, B., \& Bernal-García, A. (2018). The effects of service convenience and perceived quality on perceived value, satisfaction and loyalty in low-cost fitness centers. Sport M anagement Review, 21 (3), 250-262. doi: 10.1016/j.smr.2017.07.003

García-Pascual, F., Alguacil, M., \& Molina-García, N. (2020). Predicción de la lealtad de los usuarios de centros deportivos privados. Variables de gestión y bienestar subjetivo. Retos, 38 (38), 16-19. Doi: 10.47197/retos.v38i38.73345

González-Cutre, D., Sicilia, Á., \& Fernández, A. (2010). Hacia una mayor comprensión de la motivación en el ejercicio físico: Medición de la regulación integrada en el contexto español. Psicothema, 22(4), 841-847.

Hair, J. F., Black,W. C., Babin, B. J., Anderson, R. E., \&Tatham, R. L. (2006). Multivariate Data Analysis(Vol. 6). Upper Saddle River: Nueva Jersey: Pearson University Press.

Instituto Nacional de Salud Pública. (2016). Encuesta Nacional de Salud y N utrición de M edio Camino 2016. Mexico: Instituto Nacional de Salud Pública. Recuperado de https:/ /www.gob.mx/cms/uploads/attachment/file/ 209093/ENSANUT.pdf

Jiménez, R., Moreno, B., Leyton, M., \& Claver, F. (2015). Motivación y estadios de cambio para el ejercicio físico en adolescentes. Revista Latinoamericana de Psicología, 47(3), 196-204. https://doi.org/10.1016/j.rlp.2014.11.001

Leyton, M., Batista, M., Lobato, S., \& Jiménez, R. (2019). Validación del cuestionario del modelo transteórico del cambio de ejercicio físico. Revista Internacional De M edicina y Ciencias de la Actividad Física y del Deporte, 19(74), 329-350. Doi: 10.15366/rimcafd2019.74.010

Leyton, M., Batista, M., \& Jiménez-Castuera, R. (2020) Modelo de predicción de los estilos de vida saludables a través de la teoría de la autodeterminación de estudiantes de educación física. Revista de Psicodidáctica, 25 (1), 68-75. Doi: 10.1016/j.psicod.2019.05.002

Li, X., \& Petrick, J. F. (2010). Towards an Integrative Model of Loyalty Formation:The Role of Quality and Value. Leisure Sciences, 32(3), 201-221. Doi: 10.1080/ 01490401003709123

Markland, D., \& Tobin, V. (2004). A modification to the Behavioural Regulation in Exercise Questionnaire to 
include an assessment of amotivation. Journal of Sport \& Exercise Psychology, 26(2), 191-196. Doi:10.1123/ jsep.26.2.191

Marcus, B. H., \& Forsyth, L. H. (2009). M otivating peopleto be physically active. Champagne: Human Kinetics.

Moreno, J.A., Cervelló, E., \& Martínez, A. (2007).Validación de la Escala de Medida de los Motivos para la Actividad Física-Revisada en españoles: Diferencias por motivos de participación. Anales de psicología, 23(1), 167-176.

Mujika-Alberdi,A., Gibaja-Martins, J. J., \& García-Arrizabalaga, I. (2021). Un modelo predictivo del comportamiento de la práctica deportiva entre la generación Millennial. Retos, 39(39), 129-136. https://doi.org/10.47197/ retos.v0i39.77903

Murillo, M., Sevil, J., Abós, Á., Samper, J., Abarca-Sos, A., \& García-González, L. (2018). Análisis del compromiso deportivo de jóvenes waterpolistas: un estudio basado en la teoría de la autodeterminación. Revista Iberoamericana de Psicología del Ejercicio y el Deporte, 13(1), 111-119. Recuperado de http://www.redalyc.org/ articulo.oa?id=311153534011

Muyor, J. M., Águila, C., Sicilia,A., \& Orta, A. (2009). Análisis de la motivación autodeterminada en usuarios de centros deportivos. Revista Internacional de Medicina y Ciencias de la Actividad Física y el Deporte, 9(33), 67-80.

Nigg, C. R., \& Courneya, K. S. (1998).Transtheoretical Model: examining adolescent exercise behavior. The Journal of adolescent health, 22(3), 214-224. https: //doi.org/ 10.1016/S1054-139X(97)00141-9

Nuviala, A., García-Fernández, J., Bernal-García, A., GraoCruces, A., Teva-Villén, M. R., \& Pérez-Ordás, R. (2014). Adaptación y validación de la Escala de Intenciones Futuras de Comportamiento en usuarios de servicios deportivos. U niversitas Psychologica, 13(3), 1071-1082. http: / / dx.doi.org/10.11144/Javeriana. UPSY13-3.avei

Nuviala, A., Gómez-López, M., Grao-Cruces, A., GraneroGallegos, A., \& Nuviala, R. (2013). Perfiles motivacionales de usuarios de servicios deportivos públicos y privados. UniversitasPsychologica, 12(2), 421-431. https: / / doi.org/ 10.11144/Javeriana.upsy12-2.pmsd

Nuviala, A., Grao-Cruces, A., Tamayo-Fajardo, J.A., Nuviala, R.; Álvarez, J., \& Fernández-Martínez, A. (2013). Diseño y análisis del cuestionario de valoración de servicios deportivos (EPOD2) / Design and analysis of the valuation questionnaire of sports services (EPOD 2). Revista Internacional de Medicina y Ciencias de la Actividad Física y el Deportevol. 13 (51), 419-436.

Nuviala, A., Pérez-Turpin, J. A., Tamayo-Fajardo, J. A., \& Fernández-Martínez, A. (2011). School-age involvement in sport and perceived quality of sport services. Collegium antropologicum, 35(4), 1023-1029.

Oliver, R. L. (1997). Satisfaction: A Behavioral Perspective on the Consumer. New York: The McGraw-Hill Companies, Inc. Pérez-Amate, M. M. (2015). Modelo de servucción aplicado en tres centros de actividades físico-deportivas. (Tesis Doctoral). Pablo de Olavide, Sevilla (España). https: / /rio.upo.es/ xmlui/handle/10433/2351 ?show=full

Pérez-Ordás, R., Aznar-Cebamanos, M., Nuviala, R., \& Nuviala A. (2019). Evaluation of Extracurricular Sports Activities as an Educational Element for Sustainable Development in Educational Institutions. Sustainability, 11(12), 34743487. https://doi.org/10.3390/su11123474

Prochaska, J., \& DiClemente, C. (1982). Transtheoretical therapy: toward a more integrative model of change. Psychotherapy: Theory, Research, Practice, 19(3), 276-288. doi.org/10.1037/h0088437

Rodgers, W. M., Courneya, K. S., \& Bayduza, A. L. (2001). Examination of the transtheoretical model and exercise in 3 populations. American journal of health behavior, 25(1), 33-41. https:// doi.org/10.5993/ajhb.25.1.4

Rose, E.A., Parfitt, G., \&Williams, S. (2005). Exercise causality orientations, behavioural regulation for exercise and stage of change for exercise: exploring their relationships. Psychology of Sport and Exercise, 6(4), 399-414. doi: 10.1016/j.psychsport.2004.07.002.

Ryan, R. M., \& Deci, E. L. (2000). Intrinsic and extrinsic motivations: Classic definitions and new directions. Contemporary educational psychology, 25(1), 54-67. https: / /doi.org/10.1006/ceps.1999.1020

Thøgersen-Ntoumani, C., \& Ntoumanis, N. (2006). The role of self-determined motivation in the understanding of exercise-related behaviours, cognitions and physical selfevaluations. Journal of sports sciences, 24 (4), 393-404. doi: 10.1080/02640410500131670

Wilson, P. M., Rodgers, W. M., Loitz, C. C., \& Scime, G. (2006). «It's Who I Am...Really!» The Importance of Integrated Regulation in Exercise Contexts. Journal of Applied Biobehavioral Research, 11(2), 79-104. doi: 10.1111/j.1751-9861.2006.tb00021.x

World Health Organization. (ý2004)ý. Global strategy on diet, physical activity and health. World Health Organization. https: / / apps.who.int/iris/handle/ $10665 / 43035$

Yoshida, M., \& James, J. D. (2010). Customer Satisfaction With Game and Service Experiences: Antecedents and Consequences, Journal of Sport Management, 24 (3), 338361. doi: 10.1123/jsm.24.3.338

Zamarripa, J., Castillo, I., Baños, R., Delgado, M., \& Álvarez, O. (2018). Motivational Regulations Across the Stages of Change for Exercise in the General Population of Monterrey (Mexico). Frontiers in psychology, 9, 2368. https: / / doi.org/10.3389/fpsyg.2018.02368

Zamarripa, J., Ruiz-Juan, F., \& Ruiz-Risueño, J. R. (2019). El balance decisional, etapas de cambio y nivel de actividad física en estudiantes de bachillerato. Andamios, 16(39), 379401. doi: 10.29092/uacm.v16i39.688 